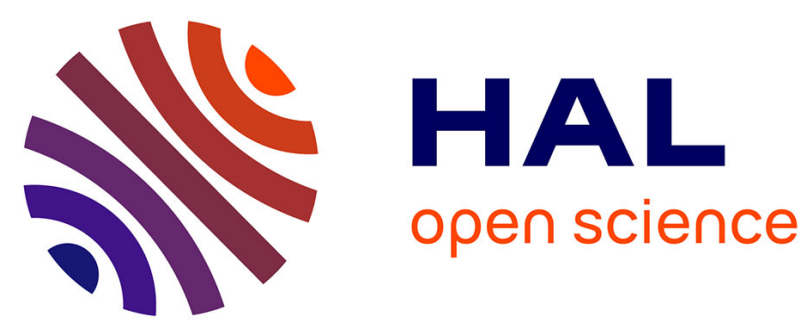

\title{
Distributed MIMO for Li-Fi: Channel Measurements, Ray Tracing and Throughput Analysis
}

Hossien B Eldeeb, Sreelal Maravanchery Mana, Volker Jungnickel, Peter

Hellwig, Jonas Hilt, Murat Uysal

\section{- To cite this version:}

Hossien B Eldeeb, Sreelal Maravanchery Mana, Volker Jungnickel, Peter Hellwig, Jonas Hilt, et al.. Distributed MIMO for Li-Fi: Channel Measurements, Ray Tracing and Throughput Analysis. IEEE Photonics Technology Letters, 2021, 33 (16), pp.916-919. 10.1109/LPT.2021.3072254 hal-03341370

\section{HAL Id: hal-03341370 \\ https://hal-amu.archives-ouvertes.fr/hal-03341370}

Submitted on 10 Sep 2021

HAL is a multi-disciplinary open access archive for the deposit and dissemination of scientific research documents, whether they are published or not. The documents may come from teaching and research institutions in France or abroad, or from public or private research centers.
L'archive ouverte pluridisciplinaire HAL, est destinée au dépôt et à la diffusion de documents scientifiques de niveau recherche, publiés ou non, émanant des établissements d'enseignement et de recherche français ou étrangers, des laboratoires publics ou privés. 


\title{
Distributed MIMO for Li-Fi: Channel Measurements, Ray Tracing and Throughput Analysis
}

\author{
Hossien B. Eldeeb ${ }^{\circledR}$, Sreelal Maravanchery Mana ${ }^{\circledR}$, Volker Jungnickel${ }^{\circledR}$, Peter Hellwig, Jonas Hilt, and \\ Murat Uysal ${ }^{\circledR}$
}

\begin{abstract}
LiFi has been considered as a promising candidate for future wireless indoor networks. The IEEE P802.15.13 and P802.11bb standardization groups agreed upon channel models generated using the non-sequential ray tracing approach of OpticStudio. In this paper, in order to validate the channel modelling approach, at first $2 \times 2$ multiple-input multiple-output (MIMO) channel measurements are carried out over $200 \mathrm{MHz}$ bandwidth using a channel sounder. The experimental scenario is also modeled in $3 \mathrm{D}$ by applying ray tracing. The obtained results indicate good agreement between simulations and measured channel impulse responses, from which parameters such as path loss and delay spread are derived. After validating the channel modeling approach, we investigate the singular values and the effect of user mobility onto the performance in a $4 \times 4$ distributed multi-user MIMO scenario.
\end{abstract}

Index Terms-Li-Fi, multiple-input multiple-output, channel measurements, ray tracing, throughput analysis.

\section{INTRODUCTION}

$\mathbf{N}$ OWADAYS, light fidelity ( $\mathrm{LiFi}$ ) is gaining increasing attention with attractive features such as operation in unregulated spectrum and robustness to electromagnetic interference [1]. Both visible light (VL) and infrared (IR) wavelengths can be used to design LiFi systems where VL may be used to offload downlink traffic while the near-IR is commonly used in the uplink for bi-directional connectivity.

Initial works on LiFi channel modeling are mainly based upon computer simulations, see e.g., the survey in [2] and references therein. In particular, non-sequential ray tracing can be used to realistically model LiFi channels. The IEEE 802.15.13 and 802.11bb standardization groups agreed upon the reference channel models generated by a non-sequential ray tracing approach [3]. This approach makes possible to obtain the channel impulse response (CIR) for complex geometries. For a precise modeling of the light propagation, it takes into account multiple reflections as well as the wavelengthdependent reflectance of the surfaces and different types of reflections (i.e. specular, diffuse, mixed).

In more recent works [4]-[6], experimental measurements of $\mathrm{LiFi}$ channels are reported. For example, in [4], a demonstration of a MIMO LiFi system using a cubic receiver has been proposed showing that cubic receiver design reduces the

This paper was presented in part at IEEE Photon. Conf. (IPC), 2020. This work was supported by the H2020 MSC ITN (VisIoN) 764461. The work of M. Uysal was supported by the Turkish Scientific and Research Council (TUBITAK) Grant 215E311. H B. Eldeeb and M. Uysal are with the Department of Electrical and Electronics Engineering, Özyeğin University, Istanbul, Turkey, 34794. S. Mana, P. Hellwig, J. Hilt, and V. Jungnickel are with the Department of Photonic Networks and Systems, Fraunhofer Heinrich Hertz Institute, Einsteinufer 37, 10587 Berlin, Germany. correlation of the MIMO channel matrix. A field measurement for $\mathrm{MIMO} \mathrm{LiFi}$ in a manufacturing cell has been reported in [5] showing that deployment of multiple distributed links allows spatial diversity and reduces the impact of shadowing and blockage to increase the robustness of $\mathrm{LiFi}$ systems. In [6], it was demonstrated that a similar setting can also be used to transport multiple data streams to multiple users in parallel. Measurements require dedicated hardware and costly equipment while characterizing LiFi channels with a realistic simulation methodology is more flexible. However, verification of the simulation approach is required, at least in few exemplary scenarios.

In an effort to validate the non-sequential ray tracing approach adopted by the IEEE, we conducted a measurement campaign in [7] and demonstrated that the results of simulations and measurements were in a good agreement. However, the considered Li-Fi scenario was limited to an empty room and single transmitter (TX) and single receiver (RX). In this letter, we extend our results to multiple-input multiple-output (MIMO) scenarios. Specifically, we consider a multiuser MIMO link between two distributed optical frontends and two mobile users in a furnished conference room [8]. Results are used to validate the ray tracing approach for MIMO and to calculate channel parameters like path loss and delay spread. Next, we investigate the singular values and the channel throughput in a modelled $4 \times 4 \mathrm{MIMO} \mathrm{LiFi}$ scenario. Moreover, we investigate the effect of user mobility on the overall throughput. The results show that the throughput depends on singular values and the SNR of each user at its individual position.

\section{Simulation Methodology}

In this section, we explain the channel modeling approach. Firstly, the 3D simulation model of the test environment (see Fig. 1.) is created in OpticStudio platform. Then, we import the CAD models of the objects (i.e., tables, tripods, chairs, etc.) inside the environment. Next, coating materials of each CAD object are defined by specifying the wavelengthdependent reflectance as well the type of reflection (i.e., specular, diffuse, and mixed). The light source specifications include total optical power, power spectral intensity, radiation pattern, and a number of emitted rays. The photodiode (PD) specifications contain sensitive area, field-of-view (FoV), and directivity. In the simulated environment, non-sequential ray tracing is performed to obtain the path length and the power of each ray emitted by the light source and absorbed by the PD. The statistics of the received photons versus their arrival time is imported into MATLAB for further processing. In this 


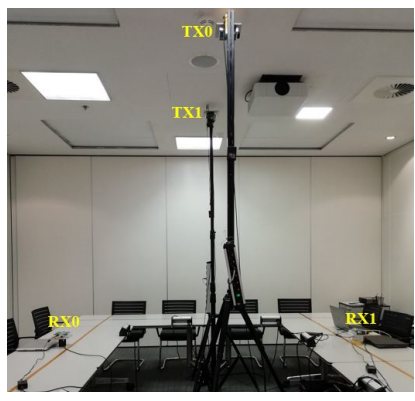

Fig. 1. Furnished conference room where measurements carried out. way, the simulated channel impulses responses (CIRs) for each link in the MIMO Li-Fi system are obtained as

$$
h_{i j}(t)=\sum_{n=1}^{N_{i j}} P_{i j}(n) \delta\left(t-\tau_{i j}(n)\right),
$$

where $N_{i j}$ is the number of rays emitted from the $j^{t h} \mathrm{TX}$ and received by the $i^{t h} \mathrm{RX}$ and $\delta$ is the Dirac delta function. For each TX $j$ and each $\operatorname{RX} i, P(n)$ and $\tau(n)$ denote the optical power and the propagation delay of the $n^{t h}$ received ray, respectively where $n=1,2, \ldots, N$. The channel frequency response (CFR) for all links can be then calculated by taking the Fourier transform (FT) of (1). After obtaining the CIR and CFR, various channel parameters can be calculated such as the path loss and the RMS delay spread [2].

\section{EXPERIMENTAL SETUP}

A channel sounder capable of measurements at frequencies of up to $250 \mathrm{MHz}$ is used for our measurements. Transmission side consist of 8-channel arbitrary waveform generator (Spectrum DN 2.662-08) which generates the waveform and feed the signals to the optical TX frontends. After the wireless transmission, signals are received at the RX frontends and fed into the connected 8-channel digitizer (Spectrum DN 2.445-08). We followed the similar experimental approach as mentioned in [5]. The power of each TX and the sensitivity of each RX are boosted by using multiple IR LEDs with same driver and multiple PDs and combining the signals after individual trans-impedance amplifiers, respectively. The TX frontend uses four OSRAM OSLON SFH-4715AS LEDs having optical power per LED of $P_{T X}=1.3 \mathrm{~W}$, centroid wavelength at $850 \mathrm{~nm}$, and half power angle of $40^{\circ}$. The RX frontend consists of five HAMAMATSU-S6968 PDs with a high sensitivity around $850 \mathrm{~nm}$ and half directivity angle of $35^{\circ}$. Since TX and RX have wide beam-width and FoV, light is transmitted into and received from many different directions, which is good for mobile scenarios. However, it also includes more non-line-of-sight signals which tend to reduce bandwidth in realistic scenarios. The waveform used in the measurement is based on direct current orthogonal frequency-division multiplexing (DC-OFDM) as described in [5]. The measured frequency response contains the response of one TX and one RX frontend. As in [7], the frontend responses calibrate out to retrieve the frequency response of the channel.

\section{VALIDATION OF RAY TRACING}

In the accompanying simulation, we consider the same measurement setup carefully by considering the radiation pattern of LEDs, the effect of the RX directivity, wavelengthdependent reflectance of walls and objects inside the room,

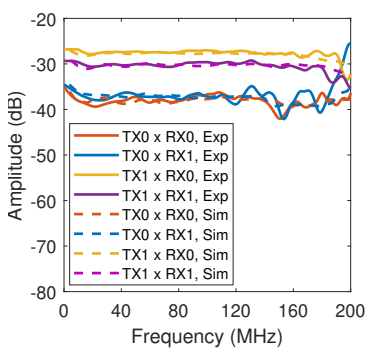

(a)

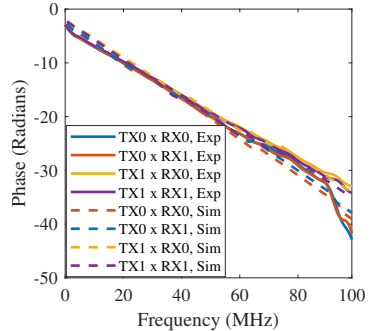

(b)
Fig. 2. Channel frequency response (CFR) of simulations and measurements (a) Amplitude response and (b) Phase response.

etc. to allow a one-to-one comparison between simulation and measurements. To validate the ray tracing, we compare both the amplitude and phase responses obtained from simulations and measurements. As shown in Fig. 1, we consider a $2 \times 2$ distributed multiuser MIMO scenario in a furnished conference room with dimensions of $(\mathrm{X} \times \mathrm{Y} \times \mathrm{Z})$ as $7.1 \mathrm{~m} \times 5.8 \mathrm{~m} \times 3 \mathrm{~m}$. Two TX frontends (TX0 and TX1) are installed at the ceiling with a height of $2.9 \mathrm{~m}$ and separated by $2 \mathrm{~m}$ with respect to $\mathrm{X}$ axis. Two users represented by two laptop devices are located on table-top height $(0.8 \mathrm{~m})$ having their corresponding $\mathrm{RX}$ frontends (RX0 and RX1) located beside. Consider the center between TX0 and TX1 is reference point $(X, Y)=(0,0)$, the locations of RX0 and RX1 are then given as $(0.65 \mathrm{~m}, 1.6 \mathrm{~m})$ and $(0.6 \mathrm{~m},-1.6 \mathrm{~m})$, respectively.

As shown in Fig. 2, the frequency responses depend on the distance between TXs and RXs. The shorter the transmission distance, the flatter is the frequency response. For TX1 (which is located $0.6 \mathrm{~m}$ closer to both RXs), the channel is nearly flat up to $150 \mathrm{MHz}$. A flat channel is obtained at lower frequencies when using TX0 but there is more ripple above $120 \mathrm{MHz}$ due to the longer transmission distance. Note, there is some ripple above $120 \mathrm{MHz}$ in the measured results which is also present in simulation results. This is attributed to multipath components, accordingly, which become more significant with respect to the line-of-sight component if distance gets larger. Hence, when considering wideband LiFi channels over longer distances, it becomes increasingly important to include the multipath effects. It is obvious from Fig. 2 that results of both the amplitude and phase responses obtained from the simulations are in a good agreement with the measurements. Considering the flat part of the CFR, i.e., frequencies below $120 \mathrm{MHz}$, the simulation results have an average difference of $0.5 \mathrm{~dB}$ with respect to the measurements.

\section{Throughrut AnAlysis}

Since these results clearly validate the simulation methodology, we can use it further to investigate more complex scenarios. We consider broadband MIMO channels at frequencies up to $100 \mathrm{MHz}$ and deploy the DC-OFDM approach with frequency points of $N_{S}$. Similar to RF, the channel is characterized based on singular value decomposition (SVD) of the MIMO channel matrix [9]. SVs represent the amplitude of the signals transmitted in parallel in a MIMO link. Generally, for a Li-Fi system with $N_{T}$ transmitters and $N_{R}$ receivers, the received signals can be represented as $\mathrm{Y}=\mathrm{HS}+\mathrm{Z}$, where $\mathrm{S}=\left(s_{1}, \cdots, s_{N_{T}}\right)^{T}$ is the transmitted 


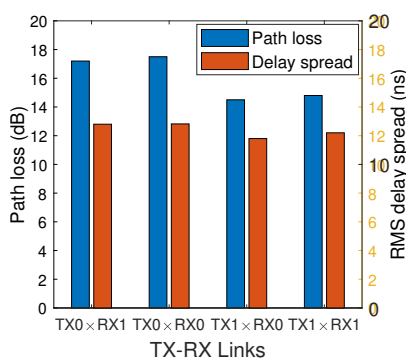

(a)

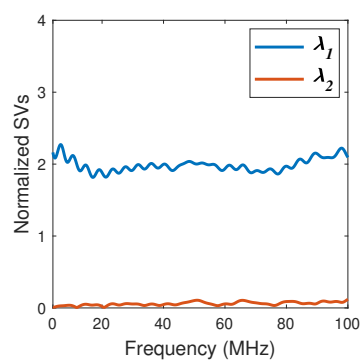

(b)
Fig. 3. Results of the $2 \times 2$ MIMO scenario (a) Path loss and RMS delay spread and (b) Normalized SVs.

signal vector, $\mathrm{Y}=\left(y_{1}, \cdots, y_{N_{R}}\right)$ is the received signal vector, and $\mathrm{Z}=\left(z_{1}, \cdots, z_{N_{R}}\right)^{T}$ is the noise vector which consists of the ambient shot and thermal noises in the receiver. $\mathrm{H}$ is MIMO channel matrix $\left(N_{R} \times N_{T}\right)$ which contains the channel coefficients between TX and RX pairs. Let $U$ and $V$ are unitary rotation matrices and assume $\Lambda$ is a quasi-diagonal "scaling" matrix. We can always decompose the channel matrix $\mathrm{H}$ by means of $\mathrm{U}, \Lambda$, and $\mathrm{V}$ as $\mathrm{H}=\mathrm{U} \Lambda \mathrm{V}^{*}$. It can be noted that both $\mathrm{H}$ and $\Lambda$ matrices have the same shape $N_{T} \times N_{R}$ and the rank is given by $\Gamma \leq \min \left(N_{T}, N_{R}\right)$. The diagonal elements of $\Lambda$ represent the real-valued and non-negative SVs of the channel matrix $\mathrm{H}$ which are given as $\left(\lambda_{1}, \cdots, \lambda_{\Gamma}\right)$. To calculate the throughput at a constant SNR of $\gamma_{0}$, we first compute the average path gain which is given by

$$
\eta_{\text {avg }}=\frac{1}{N_{T} N_{R} N_{S}} \sum_{j=1}^{N_{T}} \sum_{i=1}^{N_{R}} \sum_{k=1}^{N_{S}}\left|H_{i j k}\right|^{2} .
$$

Let $P_{r}$ is the received power at one $\mathrm{RX}$ and $P_{N o i s e}$ is the noise power. The transmitted power $\left(P_{T X}\right)$ to noise ratio is given by [10]

$$
\frac{P_{T X}}{P_{\text {Noise }}}=\frac{P_{r}}{\eta_{\text {avg }} P_{\text {Noise }}}=\frac{\gamma_{0}}{\eta_{\text {avg }}} .
$$

Finally, the channel throughput of the MIMO Li-Fi system based on DC-OFDM and SVs can be obtained by [10]

$$
C_{\text {MIMO }}=\sum_{k=1}^{N_{S}} \Delta B_{k} \cdot \sum_{j=1}^{\Gamma} \log _{2}\left(1+\frac{\gamma_{0}}{N_{T} \eta_{a v g} \zeta} \cdot \lambda_{j, k}^{2}\right),
$$

where $\Delta B_{k}=122 \mathrm{kHz}$ denotes the bandwidth occupied by $k^{t h}$ subcarrier. In (4), we include an empirical scaling factor of $\zeta=10$ to consider the impairments such as the non-linear distortions and the imperfect constellation shaping.

\section{RESUlTS AND Discussion}

\section{A. Channel Parameters}

The path loss is critical for link budget calculations and optimized system design. The results of all measured MIMO links are presented in Fig. 3(a). As expected, the corresponding path loss of both RXs with respect to TX1 (i.e., TX1-RX0 and TX1-RX1) is lower than the corresponding path loss to TX0. For example, RX1 is located $0.6 \mathrm{~m}$ closer to TX1. The path loss values are given as $14.8 \mathrm{~dB}$ and $17.2 \mathrm{~dB}$ with respect to $\mathrm{TX} 1$ and $\mathrm{TX} 0$, respectively, i.e. the longer the propagation distance, the larger is the path loss. In furnished rooms, multiple copies of the signal reach the receiver which cause different paths with different power levels and different delays. The temporal dispersion is characterized by the RMS

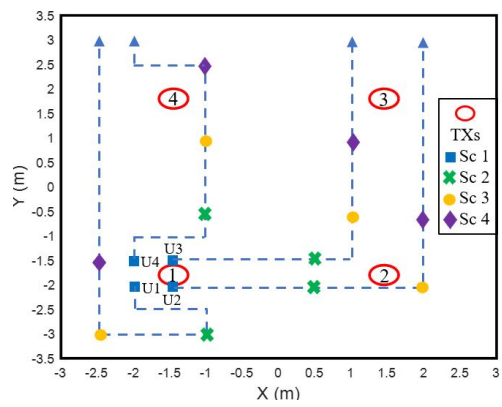

Fig. 4. MIMO Scenarios under consideration.

delay spread. It is plotted for each link in Fig. 3(a). The RMS values are given as $12.1 \mathrm{~ns}$ and $12.8 \mathrm{~ns}$ for TX1-RX1 and TX0$\mathrm{RX} 1$, respectively. Obviously, the delay spread is correlated with the path loss, i.e., higher path loss implies higher delay spread. Note that RMS values are limited by the limited of the channel sounder, which widens all pulses in the CIR.

\section{B. Singular Values}

In Fig. 3(b), at first, we investigate the SVs of the $2 \times 2$ MIMO scenario considered in the measurements (i.e., $N_{R}=$ $N_{T}=\Gamma=2$ ). There is only one strong SV, i.e. only one data stream can be transmitted in the same time slot, at least for low SNR. The main reason is that TX1 was nearer to RX1 and $\mathrm{RX} 2$ so that the corresponding column in the channel matrix dominates the result. Nonetheless, because the methodology is validated in Section IV, now we can investigate more flexible Txs and RXs positions by means of channel modeling and learn more about more complex MIMO scenarios. As shown in Fig. 4, therefore we consider a $4 \times 4 \mathrm{MIMO} \mathrm{LiFi} \mathrm{setup} \mathrm{(i.e.,}$ $N_{R}=N_{T}=\Gamma=4$ ) in a furnished conference room. The four TXs are installed near the ceiling at $2.9 \mathrm{~m}$ height uniformly distributed in the room in order to achieve homogeneous coverage. The users are kept at the table-top height facing towards the ceiling. We further consider that users are moving along specified trajectories inside the room with a step size of $0.5 \mathrm{~m}$. In Fig. 4, we investigate the SVs for specific user positions denoted by Sc 1, Sc 2, Sc 3, and Sc 4. In Sc 1, the four users (i.e., U1, U2, U3, and U4) are located at a room corner near TX1 with short separation of $0.5 \mathrm{~m}$ between each other. In Sc 2, the users become more separated, so that two users (i.e., U1 and U4) are located near TX1 while the other two users (i.e., U2 and U3) are near TX2. In Sc 3, U4 moves away from TX1 but nearer to TX4. Finally, in Sc 4, each user moves near to a different TX as shown in Fig. 4.

The singular values $\left(\lambda_{1}, \lambda_{2}, \lambda_{3}, \lambda_{4}\right)$ are calculated at each frequency bin up to $100 \mathrm{MHz}$ as shown in Fig. 5. In Sc 1 (see Fig. 5(a)), there is only one strong SV allowing only one data stream to be transmitted in the same time slot. In Sc 2 (see Fig. 5(b)), there are two strong SVs i.e. two parallel data streams are supported. In Sc 3 (see Fig. 5(c)), three strong SVs are observed. In Sc 4 (see Fig. 5(d)), a full rank of the MIMO channel matrix is achieved with four strong SVs. The results in Fig. 5 show that the principal experimental findings in [6] can be confirmed by means of ray tracing.

\section{Throughput}

The achievable throughput for the four MIMO Li-Fi scenarios versus the SNR is shown in Fig. 6(a), where SNR is 


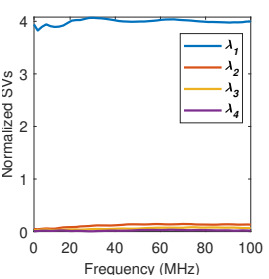

(a)

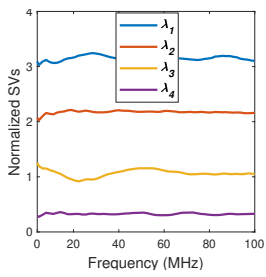

(c)

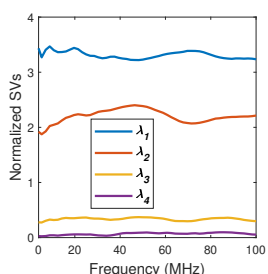

(b)

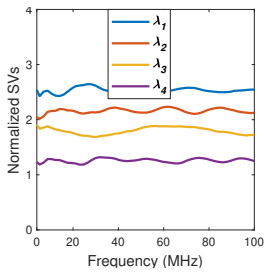

(d)
Fig. 5. Normalized SVs for (a) Sc 1, (b) Sc 2, (c) Sc 3, and (d) Sc 4.

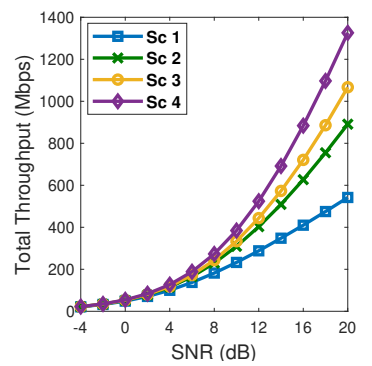

(a)

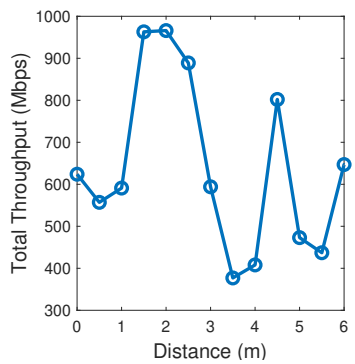

(b)
Fig. 6. (a) Total channel throughput versus SNR for Sc1, Sc 2, Sc 3, and Sc 4 and (b) Total channel throughput versus distance along trajectories.

considered constant, i.e., Foschini's formula (4) is used. It is observed that increasing the number of parallel streams results in a steeper slope of throughput particularly at high SNR. The throughput is significantly higher at any SNR if the channel allows spatial multiplexing. The throughput in Sc 1, Sc 2, Sc 3, and Sc 4 is given as 233, 309, 334, and $385 \mathrm{Mbit} / \mathrm{s}$ at $\mathrm{SNR}=10 \mathrm{~dB}$ and $542,891,1.070$ and $1.320 \mathrm{Mbit} / \mathrm{s}$ at $\mathrm{SNR}=20$ $\mathrm{dB}$, respectively.

Next, we consider the mobility of the users. If users are moving along the specified trajectories in Fig. 4, this implies variations on the optical path loss what has to be included in the throughput results. Thus, the assumption of fixed SNR becomes invalid. Therefore, we utilize the fundamental throughput formula [10, Eq.(2)] to evaluate the throughput results keeping only $P_{T X}$ as a fixed value. Fig. 6(b) illustrates the effect of mobility, where we present the channel throughput versus distance (i.e., moving steps along the trajectories). These results reveal that the user mobility creates noticeable variation of the throughput. Decrease and increase of the throughput are observed if users meet near TXs and when they are mode separated, respectively. The latter case yields more significant SVs available for spatial multiplexing. For example, at the beginning (i.e., distance $\leq 1 \mathrm{~m}$ ), only a small throughput is possible because all users locate around TX1. With only one moving step (i.e., distance of $1.5 \mathrm{~m}$ ), an increase of $360 \mathrm{Mb} / \mathrm{s}$ of the throughput is observed, because U2 and U3 move closer to TX2 allowing the transmission of two parallel data streams. Another example, when the distance increases from $4 \mathrm{~m}$ to 4.5 , the throughput climbs up by $396 \mathrm{Mb} / \mathrm{s}$ as 3 strong SVs become available. We notice that the throughput at $1.5 \mathrm{~m}$ with $2 \mathrm{SVs}$ is $160 \mathrm{Mb} / \mathrm{s}$ higher than at $4.5 \mathrm{~m}$ which has $3 \mathrm{SVs}$. This is attributed to the reduced SNR when users move farther away from the TXs. Therefore, in the real-world MIMO LiFi network, it is important to take into account the possible number of parallel data streams and the SNR in order to maximize the throughput. Besides the sum throughput considered here, advanced schedulers will take other metrics, like individual user rates, fairness among the users, latency and robustness into account. These objectives are beyond the scope of this paper and would be interesting for future research.

\section{CONCLUSIONS}

In this letter, we have used an experimental study of MIMO channels for LiFi to validate the channel models produced by non-sequential raytracing. Furthermore, we have investigated the performance. First, the measured channels have been compared to modelled ones between all TXs and all RXs. Results indicate a very good match for both, amplitude and phase responses over a wide bandwidth and confirm that raytracing creates very realistic LiFi channel models also when using MIMO with distributed optical frontends and multiple mobile users. We have analyzed the path loss and delay spread which show the well-known correlation, i.e. high path loss implies longer delay spread due to the diffuse multi-paths. Next, we have considered the performance of distributed MIMO links by means of the singular values and the achievable throughput. It was confirmed that the position of users has an essential impact on the singular values. The more distant the users are, the more significant singular values are observed, and the more streams can be supported in parallel. Finally, we investigated the effect of user mobility on the total throughput which is related to the singular values and the SNR of each user.

\section{REFERENCES}

[1] Z. Ghassemlooy et al., "Emerging optical wireless communicationsadvances and challenges," IEEE JSAC, vol. 33, no. 9, pp. 1738-1749, 2015.

[2] A. Al-Kinani et al., "Optical wireless communication channel measurements and models," IEEE Commun. Surv. \& Tut., vol. 20, no. 3, pp. 1939-1962, 2018.

[3] M. Uysal et al., "IEEE 802.15. 7r1 reference channel models for visible light communications," IEEE Commun. Mag., vol. 55, no. 1, pp. 212217, 2017.

[4] L. Wei et al., "Experimental demonstration of a cubic-receiver-based MIMO visible light communication system," IEEE Photon. J., vol. 9, no. 1, pp. 1-7, 2016.

[5] P. W. Berenguer et al., "Optical wireless MIMO experiments in an industrial environment," IEEE JSAC, vol. 36, no. 1, pp. 185-193, 2017.

[6] S. M. Mana et al., "Distributed MIMO experiments for $\mathrm{LiFi}$ in a conference room," in 12th CSNDSP. IEEE, 2020, pp. 1-5.

[7] H. B. Eldeeb et al., "Channel modelling for light communications: Validation of ray tracing by measurements," in 12th CSNDSP. IEEE, 2020, pp. 1-6.

[8] — "Channel measurements and ray tracing simulations for mimo light communication at $200 \mathrm{MHz}$,' in 2020 IPC. IEEE, 2020, pp. 1-2.

[9] A. Chaaban et al., "Capacity bounds and high-SNR capacity of MIMO intensity-modulation optical channels," IEEE Trans. Wireless Commun., vol. 17, no. 5, pp. 3003-3017, 2018.

[10] V. Jungnickel et al., "Capacity measurements in a cooperative MIMO network," IEEE trans. veh. technol., vol. 58, no. 5, pp. 2392-2405, 2008. 